\title{
A facile entry into a novel class of dispiroheterocycles through 1,3- dipolar cycloaddition
}

\author{
Jayadevan Jayashankaran, Rathna Durga R.S Manian, and Raghavachary Raghunathan* \\ Department of Organic Chemistry, University of Madras, Guindy campus, Chennai- 25, India \\ E-mail: ragharaghunathan@yahoo.com
}

\section{Dedicated to Professor S. Swaminathan, Emeritus Professor, Department of Organic Chemistry, University of Madras on his $8^{\text {th }}$ birthday \\ (received 30 Aug 04; accepted 28 Oct 04; published on the web 04 Nov 04)}

\begin{abstract}
The cycloaddition reaction of non-stabilized azomethine ylides, generated through decarboxylation and deprotonation, with $(\boldsymbol{E})$-2-arylidene-1-tetralones as dipolarophile has been investigated. A high degree of regioselectivity has been observed in the synthesis of a new class of functionalised dispiroheterocyclic compounds bearing a tetralone, acenapthenequinone and oxindole framework.
\end{abstract}

Keywords: Tetralone, azomethine ylide, dispiroheterocycles

\section{Introduction}

Spiro compounds represent an important class of naturally occurring substances characterized by highly pronounced biological properties. ${ }^{1-3}$ The most developed avenue for the synthesis of these compounds depends on the cycloaddition to an exocyclic bond. ${ }^{4-6}$

Although highly substituted spiropyrrolidines are known, there seems to be no report on the synthesis of dispiro substituted pyrrolidine heterocycles. 1,3-Dipolar cycloaddition provides a way for the synthesis of many dispiroheterocycles through the cycloaddition reaction of nonstabilised azomethine ylides with the olefinic dipolarophiles. Highly substituted pyrrolidines have attracted much interest as they contribute the central structural element of many alkaloids and pharmacological active compounds. ${ }^{7,8}$

As a part of our study ${ }^{9,10}$ on the synthesis of novel dispiropyrrolidinyl derivatives we have examined the 1,3-dipolar cycloaddition reaction of E-2-arylidene-1-tetralones with the azomethine ylide generated through a decarboxylation and deprotonation method. 


\section{Results and Discussion}

\section{Decarboxylative method}

The 1,3-dipolar cycloaddition reactions of E-2-arylidene-1-tetralones with non-stabilized azomethine ylides, generated by decarboxylative condensation of the bifunctional ketone, acenaphthenequinone, with secondary amino acids, gave a series of novel dispiropyrrolidinyl derivatives in good yield.

E-2-arylidene-1-tetralones are conformationally restricted s-cis enones which readily cycloadd to the non-stabilized ylides generated in situ by the decarboxylative condensation of acenapthenequinone $\mathbf{1}$ and sarcosine 2to afford dispiropyrrolidinyl derivatives, $1^{\prime}, 2^{\prime}, 3^{\prime}, 4^{\prime}$ tetrahydronaphthalen-1'-one-spiro[3'.3] $\mathrm{N}$-methyl-(4-aryl)-pyrrolidine-2-spiro-2"'-acenaphthen$1^{\prime \prime}$-ones 4a-e in a highly regioselective manner (Scheme 1).

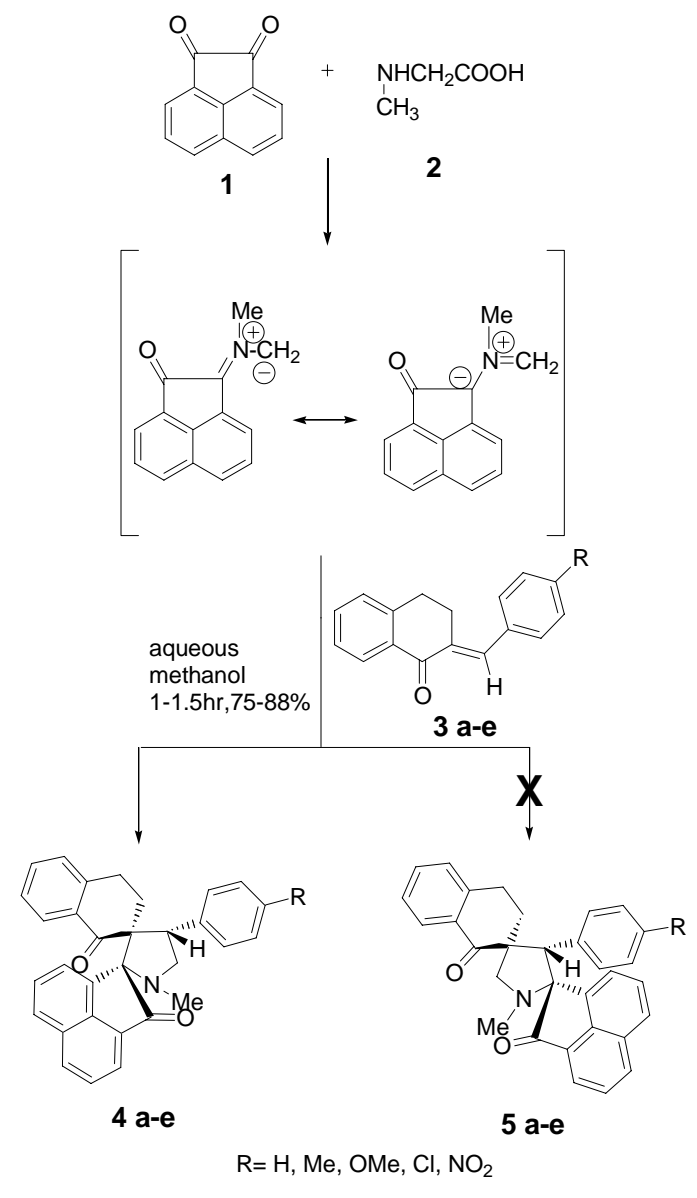

\section{Scheme 1}

The dispiroheterocyclic ring structures of products 4a-e were confirmed by $\mathrm{IR},{ }^{1} \mathrm{H} /{ }^{13} \mathrm{C}$ NMR and mass spectral studies. The IR spectrum of $\mathbf{4 a}$ showed two peaks corresponding to tetralone and acenapthenequinone ring carbonyls at 1670.9 and $1714.2 \mathrm{~cm}^{-1}$, respectively. The NMR 
spectrum of the cycloadduct $\mathbf{4 a}$ exhibited a doublet of doublets at $\delta 5.17$ due to the C-4 benzylic proton of the pyrrolidine ring. The regiochemical outcome of the Azomethine ylide cycloaddition with conformationally restricted s-cis enone, 2-arylidene-1-tetralones 3a-e is probably attributed to the involvement of the anti-ylide ${ }^{11}$ in the transistion state where the exoorientation of the dipolarophile to $\mathrm{W}$-periphery of the ylide prevents the formation of syn-ylide which is not observed due to the unfavorable steric repulsions between the carbonyl oxygen of the acenaphthequinone ring and tetralone-1-one ring systems. Further, the regiochemistry of the cycloadduct $4 \mathbf{a}$ was established by the coupling pattern in its ${ }^{1} \mathrm{H}$ NMR spectrum. Also, the ${ }^{13} \mathrm{C}$ NMR showed two signals at $\delta 69.9 \mathrm{ppm}$ and $\delta 71.2 \mathrm{ppm}$ due to the spiro carbon atoms and peaks at $\delta 192.7 \mathrm{ppm}, \delta 199.6 \mathrm{ppm}$ due to the tetralone and acenaphthequinone ring carbonyls, respectively. Identical results were observed for the other derivatives irrespective of the nature of the substituents present in the arylidene moiety of the tetralone as indicated in Table 1.

Table 1. Synthesis of $1^{\prime}, 2^{\prime}, 3^{\prime}, 4^{\prime}$-Tetrahydronaphthalen-1'-one-spiro[3'.3]N-methyl-(4-aryl)pyrrolidine-2-spiro-2"'-acenaphthen-1"-ones 4a-e

\begin{tabular}{cccc}
\hline Compound & $\mathrm{R}$ & Reaction time (h) & Yield (\%) \\
\hline $4 \mathrm{a}$ & $\mathrm{H}$ & 1.5 & 82 \\
$4 \mathrm{~b}$ & $\mathrm{Me}$ & 1.0 & 72 \\
$4 \mathrm{c}$ & $\mathrm{OMe}$ & 1.3 & 76 \\
$4 \mathrm{~d}$ & $\mathrm{Cl}$ & 1.5 & 88 \\
$4 \mathrm{e}$ & $\mathrm{NO}_{2}$ & 1.2 & 75 \\
\hline
\end{tabular}

\section{Deprotonation method}

In this method the non-stabilized azomethine ylide generated by treating benzylamine 7 with isatin 6, is reacted with 2-arylidene-1-tetralones to afford a series of dispiropyrrolidinyl oxindoles in acetonitrile at ambient temperature. Condensation of benzylamine with isatin could give rise to two configurationally distinct azomethine ylides, $\mathbf{8 a}$ and $\mathbf{8 b}$ the transition state leading to the azomethine ylide $\mathbf{8 a}$ is favoured over $\mathbf{8 b}$ due to the developing steric interaction between the carbonyl moiety and the phenyl group. ${ }^{12}$ Thus, 8a preferentially interacts with dipolarophile. The azomethine ylide $8 \mathbf{a}$ so generated readily reacts with 2 -arylidene-1-tetralones to give a series of novel dispirooxindole derivatives in a regioselective manner. The above reaction gave single dispiropyrrolidinyl oxindole heterocycles in all cases, as evidenced by TLC and spectral analyses.

The reaction of the ylide with E-2-arylidene-1-tetralones afforded a series of novel dispiroheterocycles, $\quad 1^{\prime}, 2^{\prime}, 3^{\prime}, 4^{\prime}$-tetrahydronaphthalene-1'-one-spiro-[2'.3]-(4-aryl)-pyrrolidinespiro-[2.2"]-oxindole 9a-e with high regioselectivity in good yield (Scheme 2). 


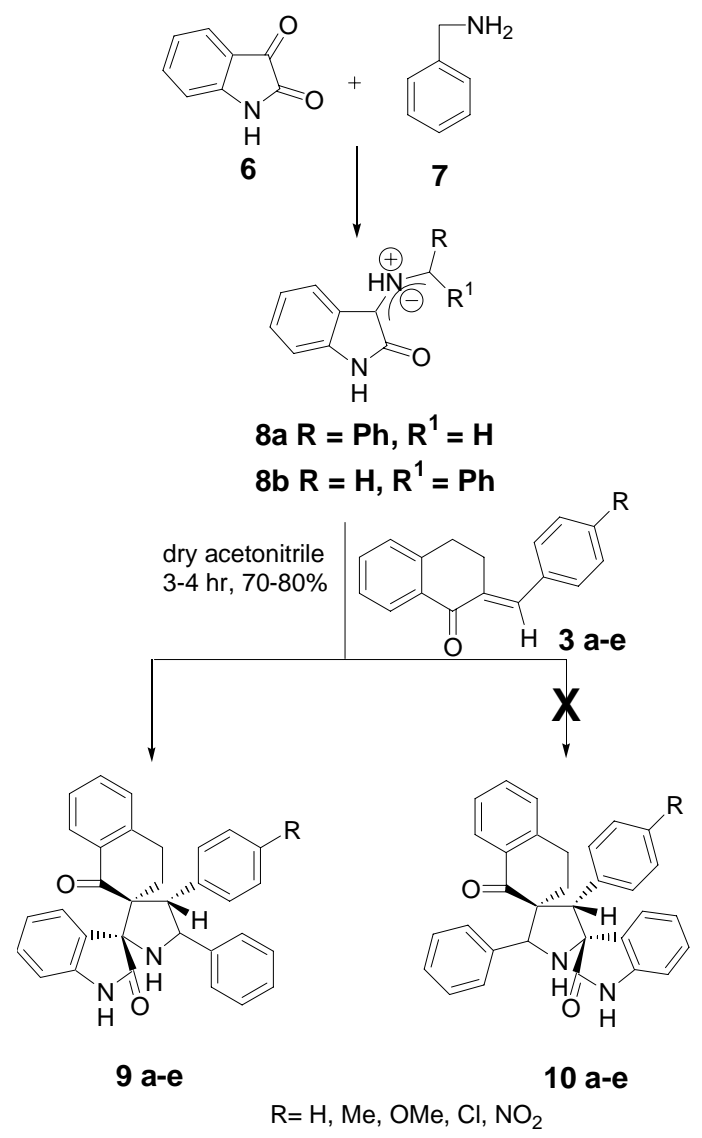

\section{Scheme 2}

The structures of the products 9a-e were confirmed by IR, ${ }^{1} \mathrm{H} /{ }^{13} \mathrm{C}$ NMR and mass spectral studies. The IR spectrum of 9a shows a peak at $1686.3 \mathrm{~cm}^{-1}$ for the tetralone carbonyl which is $10 \mathrm{~cm}^{-1}$ greater than benzylidene tetralone, which indicates the loss of conjugation. The peak at $1718.5 \mathrm{~cm}^{-1}$ confirms the presence of the oxindole moiety. The ${ }^{1} \mathrm{H}$ NMR spectrum of 9 a shows a multiplet in the region $\delta 2.44-2.76$ for the tetralone ring methylene protons. The $\mathrm{N}-\mathrm{CH}$ proton of the pyrrolidine moiety resonates as a doublet at $\delta 4.91(J=9.7 \mathrm{~Hz})$ while the NH proton of the pyrrolidine ring appears as a singlet at $\delta$ 8.3. The benzylic proton $\mathrm{H}_{\mathrm{a}}$ exhibits a peak at $\delta 5.63$ as a doublet $(J=9.7 \mathrm{~Hz})$. The ${ }^{13} \mathrm{C}$ NMR shows signals at $\delta 192.5$ and 178.2 for tetralone and oxindole ring carbonyls, respectively, which confirms the structure of the products. Identical results were observed for the other derivatives irrespective of the nature of the substituents present in the arylidene moiety of the tetralone-1-one, as indicated in Table 2.

In summary, we have studied the reactivity of s-cis restricted tetralones with two different azomethine ylides generated through decarboxylative and deprotonation methods. These studies showed that, in most cases, the azomethine cycloadditions are highly regioselective, giving good yields of novel dispiroheterocycles. These methods provide easy access to various dispiroheterocyclic frameworks, which frequently occur in alkaloids. 
Table 2. Synthesis of $1^{\prime}, 2^{\prime}, 3^{\prime}, 4^{\prime}$-Tetrahydronaphthalen-1'-one-spiro-[2'.3]-(4-aryl)-pyrrolidinespiro-[2.2"]-oxindoles 9a-e

\begin{tabular}{cccc}
\hline Compound & $\mathrm{R}$ & Reaction time (h) & Yield (\%) \\
\hline $9 \mathrm{a}$ & $\mathrm{H}$ & 3.5 & 76 \\
$9 \mathrm{~b}$ & $\mathrm{Me}$ & 3.2 & 72 \\
$9 \mathrm{c}$ & $\mathrm{OMe}$ & 4.0 & 70 \\
$9 \mathrm{~d}$ & $\mathrm{Cl}$ & 3.9 & 80 \\
$9 \mathrm{e}$ & $\mathrm{NO}_{2}$ & 3.0 & 74 \\
\hline
\end{tabular}

\section{Experimental Section}

General Procedures. All melting points are uncorrected. IR spectra were recorded on a SHIMADZU FT-IR 8300 instrument. ${ }^{1} \mathrm{H}$ and ${ }^{13} \mathrm{C}$ NMR spectra were recorded in $\mathrm{CDCl}_{3}$ using TMS as an internal standard on JEOL $400 \mathrm{MHz}$ and $100 \mathrm{MHz}$, respectively. Elemental analyses were carried out on a Perkin Elmer 250B. MS spectra were recorded on a JEOL HF 303DX spectrometer.

The starting (E)-2-arylidene-1-tetralones (3a-e) were prepared according to a literature procedure $^{12,13}$.

\section{General procedure for the synthesis of dispiroheterocycles 4a-e and 8a-e} Decarboxylative method

A solution of (E) 2-arylidene-1-tetralone 3a-e $(1 \mathrm{mmol})$, acenapthenequinone $\mathbf{1}(1 \mathrm{mmol})$ and sarcosine 2 (1 mmol) in $20 \mathrm{~mL}$ of aqueous methanol was refluxed until the disappearance of starting material as evidenced by TLC. The solvent is removed under reduced pressure and the crude product is purified by column chromatography using petroleum ether:ethyl acetate $(9: 1)$ as eluent.

\section{Deprotonation method}

A solution of $(E)$ 2-arylidene-1-tetralone $(1 \mathrm{mmol})$, isatin $(1 \mathrm{mmol})$ and benzylamine $(2 \mathrm{mmol})$ in $20 \mathrm{~mL}$ of dry acetonitrile was refluxed until the disappearance of the starting material as monitored by TLC. The solvent was then evaporated under reduced pressure and the residue was separated by column chromatography with petroleum ether-ethyl acetate (8:2) as eluent. $1^{\prime}, 2^{\prime}, 3^{\prime}, 4^{\prime}$-Tetrahydronaphthalen-1'-one-spiro[2'.3]-(4-phenyl)- $N$-methylpyrrolidinespiro[2.2"]-acenaphthen-1"-one (4a). mp: $180^{\circ} \mathrm{C}$; IR (KBr): 1670.9, $1714.2 \mathrm{~cm}^{-1} ;{ }^{1} \mathrm{H}$ NMR: $\delta$ 1.24-1.9 (m, 4H), 2.15 (s, 3H), 3.61 (dd, $J=8.6,7.4 \mathrm{~Hz}, 1 \mathrm{H}), 4.23(\mathrm{dd}, J=11.2,8.6 \mathrm{~Hz}, 1 \mathrm{H}$ ), $5.17(\mathrm{dd}, J=11.2,7.4 \mathrm{~Hz}, 1 \mathrm{H}), 6.6-8.1(\mathrm{~m}, 15 \mathrm{H}) ;{ }^{13} \mathrm{C}$ NMR: $\delta 25.9,28.7,31.2,35.6,69.9,71.2$, 74.7, 119.7, 121.6, 122.3, 122.7, 123.9, 124.0, 124.3, 125.1, 125.6, 127.5, 129.0, 130.3, 133.8, 134.5, 135.2, 135.8, 136.1, 137.2, 138.0, 139.1, 142.4, 143.9, 144.1, 192.7, 199.60; MS m/z: 
$442.9\left(\mathrm{M}^{+}\right)$; Anal. Calcd for $\mathrm{C}_{31} \mathrm{H}_{25} \mathrm{NO}_{2} \mathrm{C}$, 83.97; H, 5.64; N, 3.16. Found: C, 84.19; H, 5.8; N, 2.85 .

$1^{\prime}, 2^{\prime}, 3^{\prime}, 4^{\prime}$-Tetrahydronaphthalen-1'-one-spiro[2'.3]-(4-p-methylphenyl)- $N$-methyl pyrrolidine-spiro[2.2'"]-acenaphthen-1"'-one (4b). mp: $176^{\circ} \mathrm{C}$; IR (KBr): $1670.0,1720.0 \mathrm{~cm}^{-1}$; ${ }^{1} \mathrm{H}$ NMR: $\delta$ 1.3-2.0 (m, 4H), $2.2(\mathrm{~s}, 3 \mathrm{H}), 3.7(\mathrm{dd}, J=8.9,7.3 \mathrm{~Hz}, 1 \mathrm{H}), 4.32(\mathrm{dd}, J=11.0,8.9 \mathrm{~Hz}$, $1 \mathrm{H}), 5.0(\mathrm{dd}, J=11.0,7.3 \mathrm{~Hz}, 1 \mathrm{H}), 6.6-7.9(\mathrm{~m}, 14 \mathrm{H}) ;{ }^{13} \mathrm{C} \mathrm{NMR}: \delta 25.3,29.0,32.3,34.9,68.1$, 70.2 , 73.4, 120.4, 121.1, 123.3, 124.0, 124.8, 125.6, 126.1, 127.5, 127.8, 128.2, 128.4, 128.9, 129.0, 129.3, 130.0, 132.3, 132.7, 133.2, 135.9, 136.0, 137.4, 138.0, 198.2, 204.9; MS m/z: 457.2 $\left(\mathrm{M}^{+}\right)$; Anal. Calcd for $\mathrm{C}_{32} \mathrm{H}_{27} \mathrm{NO}_{2}$ : C, 84.03; H, 5.9; N, 3.06. Found: C, 83.84; H, 6.11; N, 2.86 .

$1^{\prime}, 2^{\prime}, 3^{\prime}, 4^{\prime}$-Tetrahydronaphthalen-1'-one-spiro[2'.3]-(4-p-methoxyphenyl)- $N$-methyl pyrrolidine-spiro[2.2']-acenaphthen-1"'-one (4c). mp: $198^{\circ} \mathrm{C}$; IR (KBr): 1668.0, $1715.6 \mathrm{~cm}^{-1}$; ${ }^{1} \mathrm{H}$ NMR: $\delta$ 1.1-2.1 (m, 4H), $2.14(\mathrm{~s}, 3 \mathrm{H}), 3.46(\mathrm{dd}, J=9.0,7.2 \mathrm{~Hz}, 1 \mathrm{H}), 3.7(\mathrm{~s}, 3 \mathrm{H}), 4.3(\mathrm{dd}, J=$ $10.8,9.0 \mathrm{~Hz}, 1 \mathrm{H}), 5.3(\mathrm{dd}, J=10.8,7.2 \mathrm{~Hz}, 1 \mathrm{H}), 6.8-7.6(\mathrm{~m}, 14 \mathrm{H}) ;{ }^{13} \mathrm{C}: \delta 26.5,30.3,33.4,35.1$, 54.9, 64.9, 72.1, 74.6, 117.3, 119.1, 120.9, 121.2, 121.8, 122.0, 122.1, 122.6, 123.7, 124.4, 125.0, 126.1, 126.4, 127.8, 128.2, 129.6, 132.7, 133.1, 133.4, 142.3, 142.7, 143.0, 143.6, 144.9, 145.0, 197.3, 199.0; MS m/z: $472.9\left(\mathrm{M}^{+}\right)$; Anal. Calcd for $\mathrm{C}_{32} \mathrm{H}_{27} \mathrm{NO}_{3}$ : C, 81.18; H, 5.71; N, 2.96 . Found: C, 81.39; H, $5.51 \mathrm{~N}, 3.04$.

$1^{\prime}, 2^{\prime}, 3^{\prime}, 4^{\prime}$-Tetrahydronaphthalen-1'-one-spiro[2'.3]-(4-p-chlorophenyl)- $N$-methyl pyrrolidine-spiro[2.2']-acenaphthen-1"'-one (4d). mp: $173^{\circ} \mathrm{C}$; IR (KBr): $1673.8,1710.4 \mathrm{~cm}^{-1}$; ${ }^{1} \mathrm{H}$ NMR: $\delta$ 1.3-2.2 (m, 4H), $2.17(\mathrm{~s}, 3 \mathrm{H}), 3.56(\mathrm{dd}, J=9.0,7.4 \mathrm{~Hz}, 1 \mathrm{H}), 4.23(\mathrm{dd}, J=11.3$, $9.0 \mathrm{~Hz}, 1 \mathrm{H}), 5.2(\mathrm{dd}, J=11.3,7.4 \mathrm{~Hz}, 1 \mathrm{H}), 6.7-7.8(\mathrm{~m}, 14 \mathrm{H}) ;{ }^{13} \mathrm{C} \mathrm{NMR}: \delta 25.7,28.1,32.6,35.9$, $68.2,72.2,75.9,120.3,121.0,121.6,122.4,122.7,123.7,124.0,124.3,124.6,124.8,125.1$, $125.6,126.0,126.9,127.1,127.8,127.9,130.1,132.6,133.5,137.2,145.6,193.7,203.1$; MS $\mathrm{m} / \mathrm{z}$ : $478\left(\mathrm{M}^{+}\right)$; Anal. Calcd for $\mathrm{C}_{31} \mathrm{H}_{24} \mathrm{NO}_{2} \mathrm{Cl}$ : C, 77.9; H, 5.02; N, 2.9. Found: C, 78.2; H, 4.82; $\mathrm{N}, 2.75$.

$1^{\prime}, 2^{\prime}, 3^{\prime}, 4^{\prime}$-Tetrahydronaphthalen-1'-one-spiro[ $\left.2^{\prime} .3\right]$-(4-p-nitrophenyl)- $N$-methyl pyrrolidine-spiro[2.2']-acenaphthen-1"'-one (4e). mp: $176^{\circ} \mathrm{C}$; IR (KBr): 1670.0, $1714.4 \mathrm{~cm}^{-1}$; ${ }^{1} \mathrm{H}$ NMR: $\delta$ 1.2-2.0 (m, 4H), $2.3(\mathrm{~s}, 3 \mathrm{H}), 3.78(\mathrm{dd}, J=8.6,7.3 \mathrm{~Hz}, 1 \mathrm{H}), 4.4(\mathrm{dd}, J=11.4,8.6 \mathrm{~Hz}$, $1 \mathrm{H}), 5.34(\mathrm{dd}, J=11.4,7.3 \mathrm{~Hz}, 1 \mathrm{H}), 6.7-8.0(\mathrm{~m}, 14 \mathrm{H}) ;{ }^{13} \mathrm{C} \mathrm{NMR}: \delta 26.3,27.8,33.0,36.1,70.2$, 71.3, 71.6, 121.3, 122.6, 122.8, 124.0, 124.3, 124.6, 124.7, 125.1, 125.3, 125.4, 125.7, 126.1, $126.2,126.7,129.0,131.1,131.9,132.0,132.8,133.5,142.1,143.7,194.4,200.9 ; \mathrm{MS}$ m/z: 488 $\left(\mathrm{M}^{+}\right)$; Anal. Calcd for $\mathrm{C}_{31} \mathrm{H}_{24} \mathrm{~N}_{2} \mathrm{O}_{4}$ : C, 76.2; H, 4.92; N, 5.74. Found: C, 76.4; H, 4.70; N, 5.94. $1^{\prime}, 2^{\prime}, 3^{\prime}, 4^{\prime}$-Tetrahydronaphthalene-1'-one-spiro-[2'.3]-(4-phenyl)-pyrrolidine-spiro-[2.2']oxindole (9a). mp: $170^{\circ} \mathrm{C}$; IR (KBr): $1686.8,1718.5 \mathrm{~cm}^{-1} ;{ }^{1} \mathrm{H}$ NMR: $\delta 2.44-2.76(\mathrm{~m}, 4 \mathrm{H}), 4.91$ $(\mathrm{d}, J=9.7 \mathrm{~Hz}, 1 \mathrm{H}), 5.63(\mathrm{~d}, J=9.7 \mathrm{~Hz}, 1 \mathrm{H}), 6.71-8.0(\mathrm{~m}, 18 \mathrm{H}), 8.49(\mathrm{~s}, 1 \mathrm{H}) ;{ }^{13} \mathrm{C} \mathrm{NMR}: \delta 25.6$, 29.3, 39.1, 49.3, 62.3, 70.3, 115.01, 115.08, 116.2, 118.9, 119.1, 120.3, 120.7, 124.3, 124.8, 125.1, 125.9, 127.3, 128.5, 130.2, 132.2, 132.4, 133.2, 140.2, 172.6, 200.5; MS m/z: $470.3\left(\mathrm{M}^{+}\right)$; Anal. Calcd for $\mathrm{C}_{32} \mathrm{H}_{26} \mathrm{~N}_{2} \mathrm{O}_{2}$ : C, 81.7; H, 5.53; N, 5.96. Found: C, 81.90; H, 5.73; N, 5.76. 
$1^{\prime}, 2^{\prime}, 3^{\prime}, 4^{\prime}$-Tetrahydronaphthalene-1'-one-spiro-[2'.3]-(4-p-methylphenyl)-pyrrolidine-spiro[2.2']-oxindole (9b). mp: $170^{\circ} \mathrm{C}$; IR (KBr): 1689.8, $1712.5 \mathrm{~cm}^{-1} ;{ }^{1} \mathrm{H}$ NMR: $\delta$ 2.2-2.6 (m, 7H), $4.82(\mathrm{~d}, J=10 \mathrm{~Hz}, 1 \mathrm{H}), 5.54(\mathrm{~d}, J=10 \mathrm{~Hz}, 1 \mathrm{H}), 6.81-7.90(\mathrm{~m}, 17 \mathrm{H}), 8.4(\mathrm{~s}, 1 \mathrm{H}) ;{ }^{13} \mathrm{C} \mathrm{NMR}: \delta$ 25.9, 27.1, 34.3, 40.2, 50.3, 68.5, 73.9,116.2, 116.8, 117.3, 119.2, 119.9, 120.2, 120.4, 121.3, 122.2, 122.9, 123.2, 125.1, 125.6, 125.8, 132.2, 132.8, 134.6, 139.2, 177.3, 201.3; MS m/z: $484.3\left(\mathrm{M}^{+}\right)$; Anal. Calcd for $\mathrm{C}_{33} \mathrm{H}_{28} \mathrm{~N}_{2} \mathrm{O}_{2}$ : C, 81.82; H, 5.78; N, 5.78. Found: C, 81.64; H, 5.99; N, 5.95 .

$1^{\prime}, 2^{\prime}, 3^{\prime}, 4^{\prime}$-Tetrahydronaphthalene-1'-one-spiro-[2'.3]-(4-p-methoxyphenyl)-pyrrolidinespiro-[2.2']-oxindole (9c). mp: $158^{\circ} \mathrm{C}$; IR (KBr): 1683.8, $1714.5 \mathrm{~cm}^{-1} ;{ }^{1} \mathrm{H}$ NMR: $\delta 2.3-2.5(\mathrm{~m}$, 4H), 3.57 (s, 3H), $5.0(\mathrm{~d}, J=9.7 \mathrm{~Hz}, 1 \mathrm{H}), 5.7(\mathrm{~d}, \mathrm{~J}=9.7 \mathrm{~Hz}, 1 \mathrm{H}), 6.6-7.8(\mathrm{~m}, 17 \mathrm{H}), 8.3(\mathrm{~s}, 1 \mathrm{H})$; ${ }^{13} \mathrm{C}$ NMR: $\delta$ 24.6, 29.3, 40.5, 42.7, 51.9, 69.9, 74.3, 117.2, 121.1, 124.2, 124.3, 127.3, 127.5, $128.3,128.9,130.2$, 132.2, 132.5, 133.7, 135.6, 138.4, 138.6, 139.7, 139.8, 140.7, 176.2, 201.2; MS m/z: $500\left(\mathrm{M}^{+}\right)$; Anal. Calcd for $\mathrm{C}_{33} \mathrm{H}_{28} \mathrm{~N}_{2} \mathrm{O}_{3}$ : C, 79.2; H, 5.6; N, 5.6. Found: C, 79.42; H, $5.26 ; \mathrm{N}, 5.8$.

1',2',3',4'-Tetrahydronaphthalene-1'-one-spiro-[2'.3]-(4-p-chlorophenyl)-pyrrolidine-spiro[2.2"']-oxindole (9d). mp: $155^{\circ} \mathrm{C}$; IR (KBr): $1685.8,1716.5 \mathrm{~cm}^{-1} ;{ }^{1} \mathrm{H}$ NMR: $\delta 2.32-2.65(\mathrm{~m}, 4 \mathrm{H})$, $4.72(\mathrm{~d}, J=9.8 \mathrm{~Hz}, 1 \mathrm{H}), 5.41(\mathrm{~d}, J=9.8 \mathrm{~Hz}, 1 \mathrm{H}), 6.57-7.5(\mathrm{~m}, 17 \mathrm{H}), 7.9(\mathrm{~s}, 1 \mathrm{H}) ;{ }^{13} \mathrm{C} \mathrm{NMR}: \delta$ 26.5, 30.3, 35.1, 54.9, 72.1, 74.6, 119.1, 121.2, 122.0, 122.6, 123.7, 125.0, 126.1, 126.3, 127.8, 128.1, 128.3, 129.7, 132.7, 133.4, 134.3, 134.7, 137.2, 141.1, 174.2, 203.6; MS m/z: $505\left(\mathrm{M}^{+}\right)$; Anal. Calcd for $\mathrm{C}_{32} \mathrm{H}_{25} \mathrm{~N}_{2} \mathrm{O}_{2} \mathrm{Cl}$ : C, 76.12; H, 4.95; N, 5.55. Found: C, 76.31; H, 4.77; N, 5.3.

$1^{\prime}, 2^{\prime}, 3^{\prime}, 4^{\prime}$-Tetrahydronaphthalene-1'-one-spiro-[2'.3]-(4-p-nitrophenyl)-pyrrolidine-spiro[2.2"']-oxindole (9e). mp: $168^{\circ} \mathrm{C}$; IR (KBr): 1683.4, $1717.2 \mathrm{~cm}^{-1} ;{ }^{1} \mathrm{H}$ NMR: $\delta 2.1-2.4(\mathrm{~m}, 4 \mathrm{H})$, $4.76(\mathrm{~d}, J=9.4 \mathrm{~Hz}, 1 \mathrm{H}), 5.73(\mathrm{~d}, J=9.4 \mathrm{~Hz}, 1 \mathrm{H}), 6.7-7.8(\mathrm{~m}, 17 \mathrm{H}), 8.1(\mathrm{~s}, 1 \mathrm{H}) ;{ }^{13} \mathrm{C} \mathrm{NMR}: \delta$ 26.8, 29.0, 37.9, 55.2, 59.2, 72.5, 120.3, 121.0, 122.4, 122.7, 123.7, 124.0, 124.6, 124.8, 125.7, $125.8,127.1,127.3,132.2,133.1,133.7,135.6,135.8,139.1,139.9,176.2,202.3 ; \mathrm{MS} \mathrm{m} / \mathrm{z}$ : $515.3\left(\mathrm{M}^{+}\right)$; Anal. Calcd for $\mathrm{C}_{32} \mathrm{H}_{25} \mathrm{~N}_{3} \mathrm{O}_{4}: \mathrm{C}, 74.56 ; \mathrm{H}, 4.85 ; \mathrm{N}, 8.15$. Found: C, 74.72; $\mathrm{H}, 4.65$; $\mathrm{N}, 8.36$.

\section{Acknowledgements}

Financial assistance from the Council for Scientific and Industrial Research is gratefully acknowledged.

\section{References}

1. Longeon,A.; Guyot,M.; Vacelet, J. Experentia 1990, 46, 548

2. Kobayashi, J.; Tsuda, M.; Agemi, K.; Shigemori, H.; Ishibashi, M.; Sasaki, T.; Mikami, Y. Tetrahedron 1991, 47, 6617. 
3. James, D. M.; Kunze, H. B.; Faulkner, D. J. J. Nat. Prod. 1991, 54, 1137.

4. Fisera, L.; Sauter, F.; Frolich, J.; Feng, Y.; Ertl, P.; Mereiter, K. Monatsh. Chem. 1994, 125, 553.

5. Fisera, L.; Sauter, F.; Frolich, J.; Feng, Y.; Ertl, P.; Mereiter, K. Monatsh. Chem. 1994, 125, 909.

6. Waldmann, H. Synlett 1995, 133.

7. Luibineau, A.; Bouchain, G.; Queneau, Y. J. Chem. Soc., Perkin Trans I 1995, 2433.

8. Deshong, P.; Leginus, J. M. J. Am. Chem. Soc. 1983, 105, 1686.

9. Manikandan, S.; Mohamed Ashraf, M.; Raghunathan, R. Synth. Commun. 2001, 31, 3593.

10. Subramaniyan, G.; Raghunathan, R. Tetrahedron 2001, 57, 2909.

11. (a) Fokas, D.; Ryan, W. J.; Casebier, D. S.; Coffen, D. Tetrahedron Lett. 1998, 39, 2235. (b) Ardill, H.; Xavier, L. R.; Grigg, R.; Montgomery, J.; Sridharan, V.; Surendrakumar, S. Tetrahedron 1990, 46, 6449.

12. Ardill, H.; Dorrity, M. J. R.; Grigg, R.; Leon-Ling, M. S.; Malone, J. F.; Sridharan, V.; Thianpatanagul, S. Tetrahedron 1990, 46, 6433.

13. Kecil, D. N.; Weitler, D.; Cromwell, N. H. J. Org. Chem. 1964, 29, 1276.

14. Mitsui, S.; Senda, Y.; Saito, H. Bull. Chem. Soc. Jpn. 1966, 39, 694. 